\title{
Escurecimento dentário e necrose pulpar após cirurgia ortognática: o laringoscópio e o traumatismo dentário
}

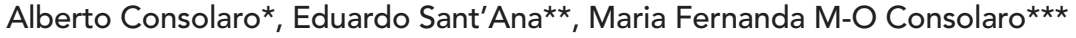

A maioria dos trabalhos sobre os efeitos da cirurgia ortognática na polpa dentária compara e ressalta que os efeitos são os mesmos encontrados em dentes submetidos ao traumatismo dentário. Estes trabalhos analisam casuísticas onde foi utilizada a osteotomia por Le Fort I, com ou sem segmentação, ou ainda da osteotomia mandibular ${ }^{16,19,23}$.

Ainda sobre os trabalhos, a grande maioria mediu as alterações do fluxo sanguíneo pulpar utilizando a fluxometria por laser Doppler ${ }^{7}$. Raramente foram realizadas análises microscópicas ${ }^{20,26,27} \mathrm{e}$ imaginológicas metodologicamente bem definidas e inquestionáveis ${ }^{8,17}$. Outra metodologia muito empregada foi o eletrodiagnóstico, para detectar a sensibilidade pulpar.

A precisão diagnóstica e a praticidade da fluxometria pulpar via laser Doppler são questionadas como metodologia mais adequada para análise de viabilidade pulpar, considerando-se que foi idealizada para tecidos moles. A presença de tecido duro poderia comprometer a sua efetividade e a precisão de resultados. Da mesma forma, são questináveis resultados obtidos apenas pelo eletrodiagnóstico pulpar.

Alguns estudos preocuparam-se em analisar a ocorrência da necrose pulpar, do escurecimento dentário e da reabsorção interna, independentemente se em dentes anteriores ou posteriores, mas há uma concentração de atenção para com os incisivos centrais superiores.

Os dentes traumatizados podem: a) manter sua vitalidade pulpar; b) sofrer necrose pulpar asséptica; c) sofrer metamorfose cálcica da polpa.

A necrose pulpar asséptica pode evoluir gradativamente para o escurecimento dentário e este sinal clínico ser a indicação de que algo está ocorrendo com o dente portador. Este sinal pode ser notado pelo paciente ou pelo profissional, ou ambos, simultaneamente.

A metamorfose cálcica da polpa foi muito conhecida clinicamente como obliteração da câmara ou do canal radicular, comumente vista em dentes traumatizados, após algumas semanas ou meses. A metamorfose cálcica da polpa ocorre quando o traumatismo lesa o feixe vásculo-nervoso, não o suficiente para impedir o fluxo sanguíneo, mas sim restringi-lo. Nestas condições de sofrimento pulpar ou estresse celular intenso por hipóxia, as células da polpa sofrem uma metaplasia, ou seja, mudam seu fenótipo e todos os fibroblastos, pe-

* Professor Titular em Patologia da FOB-USP e Professor de Pós-Graduação da FOB e FORP-USP.

** Professor Livre Docente em Cirurgia e Traumatologia da FOB-USP e Cirurgião Privado.

*** Mestre e Doutora pela FOB-USP e Ortodontista Privada - Bauru - SP. 
ricitos e células de reserva diferenciam-se aleatoriamente em odontoblastos e, de forma desorganizada, depositam uma matriz dentinária displásica no meio extracelular, ou seja uma dentina mal formada, cheias de células incluídas, a ponto de ser denominada de osteodentina.

A metamorfose cálcica da polpa pode ser simultânea em toda a polpa, mas pode ser detectada imaginologicamente ocorrendo das paredes para o centro da polpa, reduzindo gradativamente o volume pulpar a ponto de, em 6 a 12 meses, o canal estar completamente obstruído ou estreitado. Não raramente ocorre a obliteração da câmara pulpar e o estreitamento do canal radicular.

O paciente e o profissional detectam, geralmente, a metamorfose cálcica da polpa pelo escurecimento que os dentes portadores sofrem, que aumenta gradativamente à medida que a câmara pulpar se oblitera. A translucência do esmalte e a maior espessura da dentina alteram a cor do dente.

O envelhecimento pulpar pode ser acelerado por alguns fatores ambientais bucais como, por exemplo, o traumatismo dentário e a restrição de fluxo sanguíneo pulpar. O envelhecimento pulpar acelerado caracteriza-se pela redução do volume pulpar e pela deposição mais acelerada da dentina secundária, que também pode ser conhecida como dentina reacional. Da mesma forma, a freqüência dos nódulos pulpares aumenta, inclusive do ponto de vista radiográfico. Microscopicamente, o envelhecimento pulpar caracteriza-se, ainda, pela redução da celularidade e fibrosamento da matriz extracelular com áreas extensas de hialinização.

Ao analisar polpas de dentes de pacientes submetidos a cirurgias ortognáticas, muitos trabalhos $3,4,12,13,15,22$ concluíram que há uma hiperemia logo após a osteotomia e que isto pode contribuir para alterações de cor e perda da vitalidade, embora reconheçam que outros autores obtiveram isquemia da polpa e não hiperemia em suas casuísticas analisadas ${ }^{9,10,14,18,21}$. Para alguns estudiosos, as alterações pulpares são insignificantes ${ }^{6,11,24}$. Enfim, apesar de utilizarem a mesma metodologia, na flu- xometria com laser Doppler não há uniformidade sobre as alterações de fluxo sanguíneo pulpar, possivelmente induzidas pela cirurgia ortognática. Os períodos de análise variam entre 1, 7, 14 e 30 dias, passando para 3, 6 e 12 meses após transcorrida a cirurgia.

Ainda analisando a literatura pertinente como um todo, as alterações pulpares encontradas no fluxo sanguíneo são consideradas reversíveis ao longo das semanas subseqüentes à cirurgia, restabelecendo-se a normalidade. Os resultados de Harada et al. ${ }^{12,13}$ e Sato et al. ${ }^{22}$ revelaram que 1 a 5 dias é o período de latência e que depois de 6 dias a 3 meses o fluxo sanguíneo pulpar vai voltando ao normal. Nos casos em que o fluxo sanguíneo pulpar não foi restabelecido na plenitude da situa-

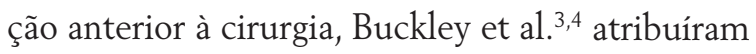
este fato à redução do volume pulpar, decorrente de um envelhecimento pulpar acelerado e induzido, caracterizado pela deposição aumentada de dentina secundária ou reacional. Vedtofte et al. ${ }^{26}$, em cirurgias ortognáticas experimentais similares em macacos, encontraram esta redução do volume pulpar em apenas 2,3\% do dentes e após 28 meses.

Em relação à perda da sensibilidade pulpar, os resultados de Harada et al. ${ }^{12,13}$ e Sato et al. ${ }^{22}$ revelaram que a sensibilidade negativa perdura até 14 dias depois da cirurgia, mas 3 meses depois a sensibilidade volta ao normal em $90,9 \%$ dos dentes. Por outro lado, De Jongh et al..$^{5}$ observaram que 6 a $29 \%$ dos dentes têm perda de sensibilidade por até 54 meses após a cirurgia ortognática.

Nos centros cirúrgicos, há uma rotina médica padronizada em função dos riscos e conseqüências a que o paciente a ser operado se submete, incluindo-se os procedimentos anestesiológicos, os controles das funções vitais e as manobras para propiciar à equipe cirúrgica o acesso necessário ao campo operatório.

Durante os processos de anestesia e intubação dos pacientes, vários instrumentos e materiais devem passar pela boca, incluindo o laringoscópio 


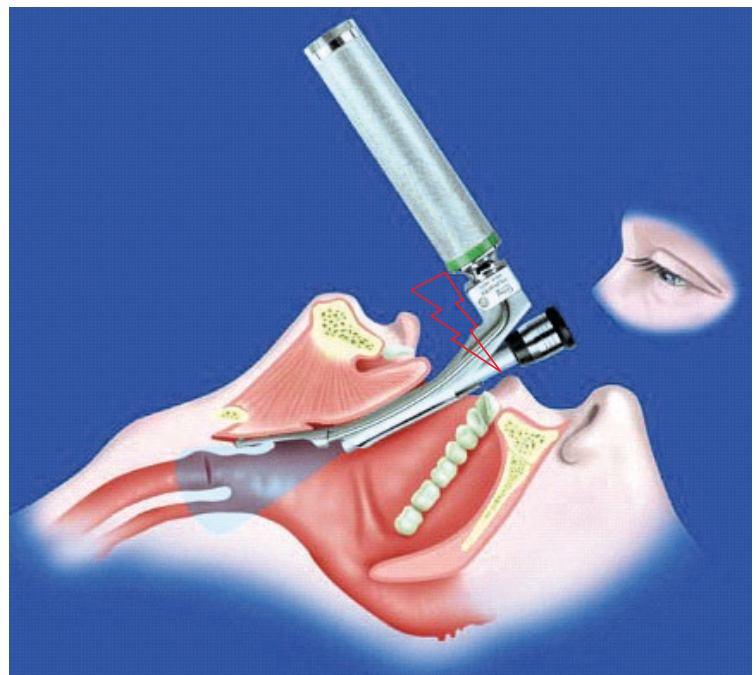

FIGURA 1 - A utilização do laringoscópio nos procedimentos de anestesia e preparo do paciente para intubação durante a cirurgia pode levar acidentalmente (seta) ao traumatismo dentário, especialmente nos incisivos superiores. FONTE: http://img.alibaba.com/photo/10949856/Truview_Optical_View_Laringoscope.jpg.

(Fig. 1). Este robusto aparelho, necessariamente, abaixa a língua e os tecidos moles da orofaringe para se observar a laringe e, ao mesmo tempo, propiciar condições necessárias para a intubação. Neste processo de manipulação e preparo da boca, orofaringe e laringe, para se anestesiar e controlar as funções vitais do paciente por parte do anestesiologista, a possibilidade de traumatismo dentário é elevada (Fig. 1). Desta forma, o contato do laringoscópio e a colocação e retirada de artefatos referentes à intubação podem promover, acidentalmente, luxação dentária, intrusão, fraturas coronárias e/ou radiculares e até avulsão dentária. Como o paciente está anestesiado ou sedado e a prioridade momentânea refere-se a dar condições de operar o paciente, a maioria dos traumatismos dentários não é imediatamente detectada. As suas conseqüências são diagnosticadas apenas depois de alguns dias, semanas ou meses. Entre estas conseqüências estão o escurecimento por metamorfose cálcica da polpa, necrose pulpar, perda de sensibilidade, mobilidade dentária excessiva e outras.

Quando o cirurgião secciona a maxila por Le Fort I, com ou sem segmentação, a linha de oste- otomia geralmente é suficientemente alta e longe da porção apical dos dentes, a ponto de evitar a perda de suprimento sanguíneo dos dentes superiores. Em casos em que esta linha de osteotomia for muito próxima da região apical, é natural se esperar que alguns dentes sofram metamorfose cálcica da polpa, envelhecimento acelerado e até necrose pulpar, mas não apenas em um dente, como em geral se observa. Em casos de restrição de suprimento sanguíneo, os tecidos periodontais, incluindo a gengiva, também sofrem conseqüências como a recessão gengival e perda de crista óssea. Quando um único dente apresenta uma destas alterações, a possibilidade de traumatismo dentário transoperatório aumenta consideravelmente.

A cirurgia ortognática foi descrita inicialmente por Blair ${ }^{1}$, em 1907, e hoje é rotineiramente utilizada em várias partes do mundo para corrigir as discrepâncias dentofaciais. Em síntese, os escurecimentos e a perda de sensibilidade dentária após cirurgias ortognáticas requerem atenção e cuidados. Quando ocorrem, para esclerecer sua verdadeira causa e origem, alguns questionamentos devem ser realizados, como por exemplo:

1) quantos dentes estão afetados?

2) há fraturas de esmalte ou trincas acentuadas associadas?

3) radiograficamente, a câmara pulpar e o canal radicular estão obstruídos?

4) há perda de sensibilidade pulpar?

5) definitivamente não há fratura radicular?

6) qual o grau de mobilidade dentária presente?

7) os tecidos periodontais do mesmo dente estão comprometidos?

Os trabalhos detalhados sobre a situação dentária dos pacientes submetidos a cirurgias ortognáticas, quanto ao escurecimento dentário, perda de sensibilidade dentinária e necrose pulpar, são $\operatorname{raros}^{2,3,4,16,25,26}$. Há pouca explicação com base biológica aplicada ao conhecimento endodôntico e sobre os efeitos teciduais do traumatismo dentário. Os aspectos clínicos e imaginológicos são mui- 
to pouco explorados no contexto da biologia da polpa e do diagnóstico endodôntico.

Os achados relatados na literatura e os encontrados na prática clínica sobre os dentes escurecidos, perda de sensibilidade e necrose pulpar após a cirurgia ortognática têm muito em comum com os observados em dentes submetidos a traumatismos. Novas pesquisas, com designs apropriados e resultados bem interpretados, são necessárias e os insights devem levar em consideração também a observação dos procedimentos transoperatórios da cirurgia ortognática.

\section{REFERÊNCIAS}

1. BLAIR, V. P. Operations on jaw bones and face: study of a etiology and pathological anatomy of developmental malrelations of maxilla and mandible to each other and to facial outline and of operative treatment when beyond the scope of the orthodontist. Gynecol. Obstet., [s.I.], v. 4, p. 67-68, 1907.

2. BROWNE, R. M.; BRADY, C. L.; FRAME, J. W. Tooth pulp changes following Le Fort I maxillary osteotomy in a primate model. Br. J. Oral Maxillofac. Surg., Edinburgh, v. 28, p. 1-7, 1990.

3. BUCKLEY, J. G. An evaluation of the changes in pulpar blood flow associated with orthognathic surgery. 1994 Dissertation (M.Sc.D.)-University of Wales Library, Aberystwyth, 1994.

4. BUCKLEY J. G. et al. An evaluation of the changes in maxillary pulpal blood flow associated with orthognathic surgery. Br. J. Orthod., Oxford, v. 26, no. 1, p. 39-45, Mar. 1999.

5. DE JONGH, M.; BARNARD, D.; BIRNIE, D. Sensory nerve morbidity following Le Fort I osteotomy. J. Oral Maxillofac. Surg., Philadelphia, v. 14, p. 10-13, 1986.

6. DI, S. et al. Long-term evaluation of human teeth after Le Fort I osteotomy: a histologic and developmental study. Oral Surg. Oral Med. Oral Pathol., St. Louis, v. 65, p. 379386, 1988.

7. DICERBO, M. A. Blood flow determined by laser Doppler flowmetry in orthognathic surgery. Am. J. Orthod. Dentofacial Orthop., St. Louis, v. 103, p. 488, 1993.

8. ELLINGSEN, R. H.; ARTUN, J. Pulpal response to orthognathic surgery: a long-term radiographic study. Am. J. Orthod. Dentofacial Orthop., St. Louis, v. 103, no. 4, p. 338-343, Apr. 1993.

9. EMSHOFF, R.; KRANEWITTER, R.; NORER, B. Effect of Le Fort I osteotomy on maxillary tooth-type-related pulpar blood-flow characteristics. Oral Surg. Oral Med. Oral Pathol. Oral Radiol. Endod., St. Louis, v. 89, no. 1, p. 8890, Jan. 2000.

10. EMSHOFF, R. et al. Effect of segmental Le Fort I osteotomy on maxillary tooth-type-related pulpar blood-flow characteristics. Oral Surg. Oral Med. Oral Pathol. Oral Radiol. Endod., St. Louis, v. 89, no. 6, p. 749-752, June 2000.

11. GEYLIKMAN, Y. B. et al. Effect of Le Fort I osteotomy on human gingival and pulp circulation. Int. J. Oral Maxillofac. Surg., Copenhagen, v. 24, p. 255-260, 1995.

12. HARADA, K.; SATO, M.; OMURA, K. Blood-flow and neurosensory changes in the maxillary dental pulp after differing Le Fort I osteotomies. Oral Surg. Oral Med. Oral Pathol. Oral Radiol. Endod., St. Louis, v. 97, no. 1, p. 12-17, Jan. 2004.
13. HARADA, K.; SATO, M.; OMURA, K. Blood-flow change and recovery of sensibility in the maxillary dental pulp during and after maxillary distraction: a pilot study. Oral Surg. Oral Med. Oral Pathol. Oral Radiol. Endod., St. Louis, v. 98, no. 5, p. 528-532, Nov. 2004.

14. ILDRESANO, A. T.; LUNDELL, M. I. Blood flow changes in the rabbit maxilla following an anterior osteotomy. J. Dental Res. Alexandria, v. 62, p. 743-735, 1983.

15. JUSTUS, T. et al. Human gingival and pulpar blood flow during healing after Le Fort I osteotomy. J. Oral Maxillofac. Surg., Philadelphia, v. 59, p. 2-7, 2001.

16. LANIGAM, D. T.; HEY, J. WEST, R. Aseptic necrosis following maxillary osteotomies, report of 36 cases. J. Oral Maxillofac. Surg., Philadelphia, v. 48, p. 142-156, 1990.

17. LEIBOLD, D. G.; TILSON, H. B.; RASK, K. R. A subjective evaluation of the re-establishment of the neurovascular supply of teeth involved in anterior maxillary osteotomy procedures. J. Oral Surg., Chicago, v. 32, p. 531, 1971.

18. MEYER, M. W.; CAVANAUGH, G. D. Blood flow changes after orthognathic surgery: maxillary and mandibular subapical osteotomy. J. Oral Surg., Chicago, v. 34, p. 495, 1976.

19. MOL VAN OTTERLOO, J. C. et al. Intra and early post-operative complications of the Le Fort I osteotomy: a retrospective study on 410 cases. J. Craniomaxillofacial Surg., Edinburgh, v. 19, p. 217-222, 1991.

20. OHZEKI, H.; TAKAHASHI, S. Histological pulp changes in the dental osseous segment following anterior maxillary osteotomy. Bull. Tokyo Dent. Coll., Tokyo, v. 21, p. 21, 1980.

21. QUEJADA, J. G. et al. Wound healing associated with segmental total maxillary osteotomy. J. Oral Maxillofac. Surg., Philadelphia, v. 44, p. 366, 1986.

22. SATO, M. et al. Blood-flow change and recovery of sensibility in the maxillary dental pulp after a single-segment Le Fort I osteotomy. Oral Surg. Oral Med. Oral Pathol. Oral Radiol. Endod., St. Louis, v. 95, no. 6, p. 660-664, June 2003.

23. SUGG, G. R. et al. Early pulp changes after anterior maxillary osteotomy. J. Oral Surg., Chicago, v. 39, p. 14-20, 1981

24. SUMMERS, L.; BOOTH, R. The early effects of segmental surgery on the human pulp. Int. J. Oral Surg., Copenhagen, v. 4, p. 236-241, 1975.

25. VEDTOFTE, P. Pulp canal obliteration after Le Fort I osteotomy. Endod. Dent. Traumatol., Copenhagen, v. 5, p. 274-278, 1989.

26. VEDTOFTE, P.; NATTESTAD, A. Pulp sensibility and pulp necrosis after Le Fort I osteotomy. J. Craniomaxillofac. Surg., Edinburgh, v. 17, p. 167-171, 1989.

27. ZISSER, G.; GATTINGER, B. Histologic investigation of pulpal changes following maxillary and mandibular alveolar osteotomies in the dog. J. Oral Maxillofac. Surg., Philadelphia, v. 40, p. 332, 1982. 